\title{
Human leukocyte antigen-DR expression might predict outcomes in severe sepsis, but diabetes mellitus cannot be ignored
}

\author{
Jun Li ${ }^{*}$, Xianshi Zhou, Ye Ye and Guanghua Guanghua
}

See related research by Drewry et al., https://ccforum.biomedcentral.com/articles/10.1186/s13054-016-1505-0

We read with great interest, but also with some concern, the paper by Drewry et al. recently published in Critical Care [1]. In this observational study, the authors concluded that human leukocyte antigen-DR (HLA-DR) expression might be a more accurate predictor of mortality and acquisition of secondary infections than lipopolysaccharide-stimulated TNF- $\alpha$ production in critically ill patients.

The primary concern with the article is that it overlooks the effects that diabetes might exert on the outcomes. Notably, the ratio of comorbid diabetes was statistically higher in the survivors than the nonsurvivors $(P=0.034)$. Diabetes has close and complex relationship with sepsis-it can not only alter the immune response (including HLA-DR expression) [2, 3], but also influence the mortality rate and risk of infection in patients with sepsis [2]. In addition, hyperglycemia, insulin [2], and obesity [4] secondary to diabetes can also impact on both the immune system and critical outcomes. Thus, it did not seem persuasive that HLA-DR expression could completely predict outcomes without adjusting for the covariate of diabetes.

Similarly, the APACHE II and SOFA scores, which reflect the severity of the disease, were statistically lower in the survivors than the non-survivors $(P<0.001$ and $P$ $=0.009$, respectively). However, in a prospective study on mHLA-DR [5], in which the severity level based on SAPS II or SOFA score was adjusted, the original statistical relationship between early mHLA-DR downregulation and outcomes in the whole population disappeared $(P>0.05)$. Thus, it is plausible and necessary to adjust APACHE II and SOFA scores for statistical analysis in this paper.

\footnotetext{
* Correspondence: junlilijun123@126.com

Emergency Department, Guangdong Provincial Hospital of Chinese

Medicine, affiliated to Guangzhou University of Chinese Medicine, 111 Dade Road, Yuexiu District, Guangzhou 510120, China
}

The two inaccuracies described above are examples of colinearity, which suggest that not only univariate but also multivariable analysis should be carried out in this study to exclude interactions between variables and make the conclusion more prudent and accurate.

In addition, we do not understand why the APACHE II and SOFA scores were calculated without the neurological component, which can be easily done as the study was described as prospective. Moreover, we suggest that receiver operating characteristics (ROC) curves for the outcomes and these two markers be drawn to compare and determine which is the better predictive marker and what is the best, most pragmatic threshold (i.e., maximized sum of sensitivity and specificity).

We appreciate the meaningful work of Drewry et al., which provides us with a new perspective on predictive indicators in severe sepsis, though the statistical methods in the study need some amendments.

\section{Publisher's Note}

Springer Nature remains neutral with regard to jurisdictional claims in published maps and institutional affiliations.

Published online: 19 June 2017

References

1. Drewry AM, Ablordeppey EA, Murray ET, Beiter ER, Walton AH, Hall MW, Hotchkiss RS. Comparison of monocyte human leukocyte antigen-DR expression and stimulated tumor necrosis factor alpha production as outcome predictors in severe sepsis: a prospective observational study. Crit Care. 2016;20:334

2. Schuetz P, Castro P, Shapiro NI. Diabetes and Sepsis: Preclinical Findings and Clinical Relevance. Diabetes Care. 2011;34(3):771-78.

3. Hamzeh AR, Nair P, Al Ali MT. The profile of HLA-DRB1 alleles in Arabs with type 1 diabetes; meta-analyses. Hla. 2016;87(1):25-30.

4. Kuperman EF, Showalter JW, Lehman EB, Leib AE, Kraschnewski JL. The impact of obesity on sepsis mortality: a retrospective review. BMC Infect Dis. 2013;13(1):377.

5. Lukaszewicz AC, Grienay M, Resche-Rigon M, Pirracchio R, Faivre V, Boval B, Payen D. Monocytic HLA-DR expression in intensive care patients: Interest for prognosis and secondary infection prediction. Crit Care Med. 2009; 37(10):2746-52. 